\title{
HIGH PRESSURE RINSING SYSTEM COMPARISON
}

\author{
D. Sertore*, M. Fusetti, P. Michelato, C. Pagani (INFN Milano - LASA, Segrate (MI), Italy), \\ T. Higo, J. Hong, K. Saito (KEK, Tsukuba, Japan), \\ G. Ciovati, T. Rothgeb (JLAB, Newport News, USA).
}

\begin{abstract}
High pressure rinsing (HPR) is a key process for the surface preparation of high field superconducting cavities. A portable apparatus for the water jet characterization, based on the transferred momentum between the water jet and a load cell, has been used in different laboratories. This apparatus allows collecting quantitative parameters that characterize the HPR water jet. In this paper, we present a quantitative comparison of the different water jets produced by various nozzles routinely used in different laboratories for the HPR process.
\end{abstract}

\section{INTRODUCTION}

The possibility to reach high surface electric field in superconducting cavities is the result of different processes applied to the resonators during their fabrication. One of the key steps is the high pressure rinsing (HPR), which removes chemical residuals and particles from the surface [1,2]. Despite the significant improvements in cavities performance after the introduction of the HPR in the cavity cleaning procedure, there is still a lack of understanding of the HPR cleaning mechanism. Moreover, different laboratories apply different parameters for the HPR (water pressure and throughput, process duration, number and type of nozzles, cavity rotating speed and jet vertical movement characteristic). While many of the HPR parameters are measured, logged and controlled during the process, the jet parameters are usually not known. The purpose of this paper is to present the characterization of the water jets used at KEK and JLAB during the HPR process.

This work is of primary importance for setting up a "standard" HPR procedure with the aim of reducing the performance spread in cavity production, the amount of ultra pure water used in the HPR and finally the cost of the cavity preparation. The information taken during these measurements, combined with HPR process parameters, should identify new significant measurable variables such as the jet power, the deposited energy on the cavity surfaces and the pressure. Those should be correlated with the quality of the cleaning process.

\section{EXPERIMENTAL SETUP}

The measuring device used for the jet characterization was developed at INFN Milano - LASA and used there to characterize different nozzles [3]. It is based on a load cell ( $2 \mathrm{~kg}$ dynamic range, resolution $1 \mathrm{~g}$ ) properly modified for its application in a wet environment. Different targets can be mounted on the load cell to intercept, at different angles, the water jet. The $90^{\circ}$ target is used for the jet qualification and jet profiling. Different tapered target are used for the study of water jet interacting at an angle with the surfaces, as during the HPR of SRF cavities.

The device is quite simple and only small modifications are needed to install it in the HPR system. The system can be used as a routine check up of the nozzle status and as a verification of the jet characteristics.

We characterized the jets of the JLAB HPR systems (R\&D and Production), and of KEK and Nomura Plating systems, where KEK cavities are processed. Measurements at DESY are foreseen in the next months. Fig. 1 shows the device installed in the JLAB Production HPR system, located inside the clean room. Table 1 summarizes the laboratory parameters during HPR characterization.

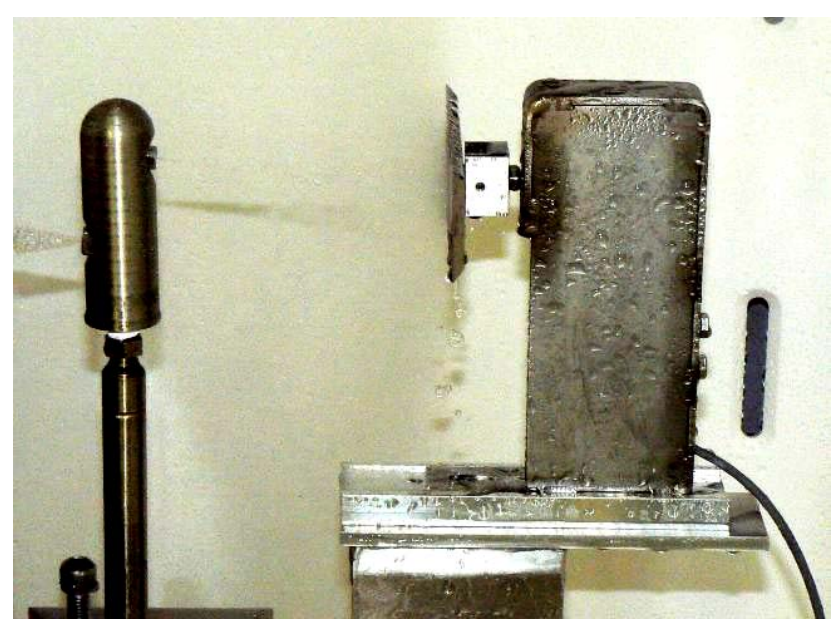

Figure 1: HPR jet characterization at JLAB.

Table 1: Laboratory parameters during HPR characterization. "Spraying System Co." nozzles generate a "fan" type jet. Sapphire nozzles and drilled hole (KEK) produce round jets.

\begin{tabular}{|c|c|c|c|c|}
\hline Lab. & $\begin{array}{c}\# \\
\text { nozzles }\end{array}$ & Tested nozzles & $\begin{array}{c}\text { Flow [1/min] } \\
(1 \text { nozzle })\end{array}$ & $\begin{array}{c}\text { Pump } \\
\text { Press } \\
{[\text { bar }]}\end{array}$ \\
\hline $\begin{array}{c}\text { JLAB } \\
\text { Prod }\end{array}$ & 2 & $\begin{array}{c}\text { Spraying System } \\
1502,4002,40015\end{array}$ & 5 @ 85 bar & 85 \\
\hline $\begin{array}{c}\text { JLAB } \\
\text { R\&D }\end{array}$ & 2 & $\begin{array}{c}\text { Spraying System } 1502 \\
\text { Sapphire } 0.4 \mathrm{~mm}\end{array}$ & 5 @ $85 \mathrm{bar}$ & 85 \\
\hline KEK & 8 & $0.6 \mathrm{~mm} \mathrm{SS}$ & $1.5 @ 70 \mathrm{bar}$ & $70-50$ \\
Ibaraki & 9 & $\begin{array}{c}0.6 \mathrm{~mm} \mathrm{SS} \\
0.6 \mathrm{~mm} \mathrm{SS}\end{array}$ & $\begin{array}{c}1.1 @ 50 \mathrm{bar} \\
0.9 @ 40 \mathrm{bar}\end{array}$ & $50-40$ \\
\hline $\begin{array}{c}\text { KEK } \\
\text { Nomura }\end{array}$ & 8 & \multicolumn{4}{|l}{} \\
\hline
\end{tabular}

\footnotetext{
*Daniele.Sertore@mi.infn.it
} 


\section{JET FORCE AND PROFILE}

The theoretical force exerted by a water jet on a perpendicular target is simply given by the Bernoulli law and momentum conservation [4]

$$
F=\rho \cdot Q \cdot u
$$

where $\rho$ is the water density, $\mathrm{Q}$ the water flow and $\mathrm{u}$ the jet speed given by Bernoulli (where $\mathrm{p}$ is the pressure).

$$
u=\sqrt{\frac{2 \cdot p}{\rho}}
$$

With the numbers reported in Table 1 , the expected force exerted by the jet, for the case of the KEK HPR, is $2.9 \mathrm{~N}$, while we measured $2.5 \mathrm{~N}$ : this discrepancy is due to losses in the nozzle.

Once the jet is moved out of the target, the force measured during the movement represents an integral along the direction of motion. In the normal direction we assume to have the full jet on the target. A typical measurement for a KEK round jet is shown Fig. 2, where the measurements of a new and of a used nozzle are compared. The jet produced by the used nozzle shows large pressure variations and instabilities compared to the new one.

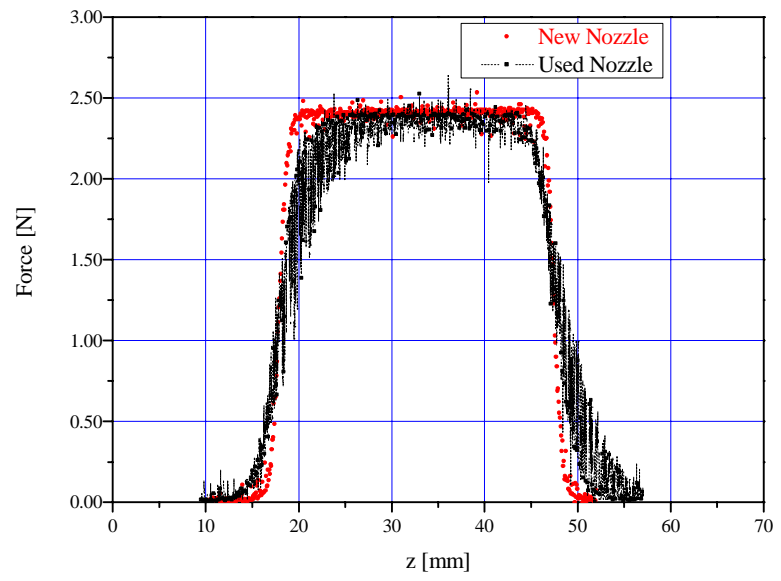

Figure 2: Force measured on the load cell for a jet produced by a new (red dots) and a used nozzle.

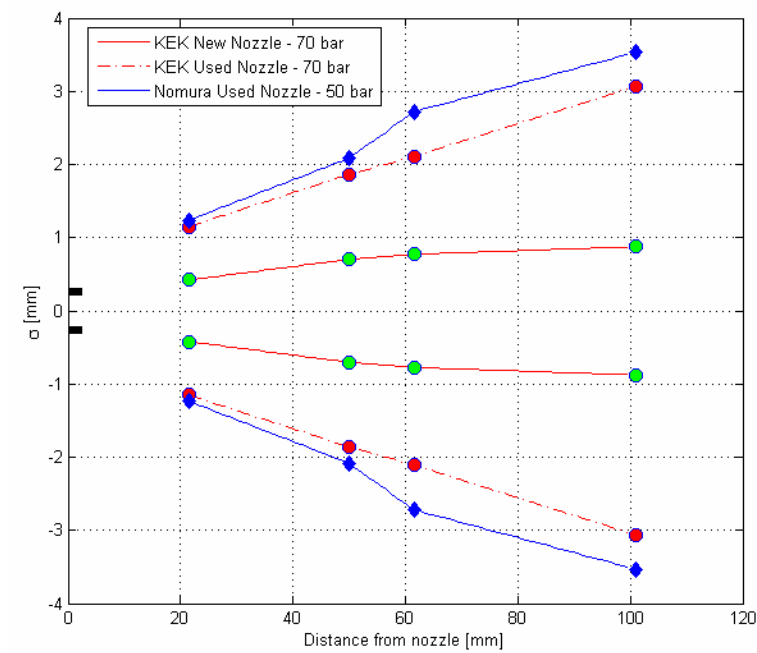

Figure 3: Change of jet sigma with distance for new and old nozzle. The jet diameter is $0.6 \mathrm{~mm}$.
For the round nozzle, we assume that the force profile has a Gaussian shape and we use it to fit the data. The calculated sigma values are shown in Fig. 3. The water jet produced by used nozzles shows a larger sigma values than the one generated by new nozzles, hint of a deterioration of the nozzles during long time operation. In the case of measurements done at Nomura, the high sigma values are also correlated with instabilities in the HPR pump. Optical and SEM images confirmed the presence of corrosion effect in the used nozzles [5, 6].

Given the profiles, the pressure exerted by a stationary jet can be easily represented at different distances from the nozzles. At $101 \mathrm{~mm}$, (about the ILC cavity equator) the pressure distribution is shown in Fig. 4, for a new nozzle and a used one. The difference in pressure is about one order of magnitude.
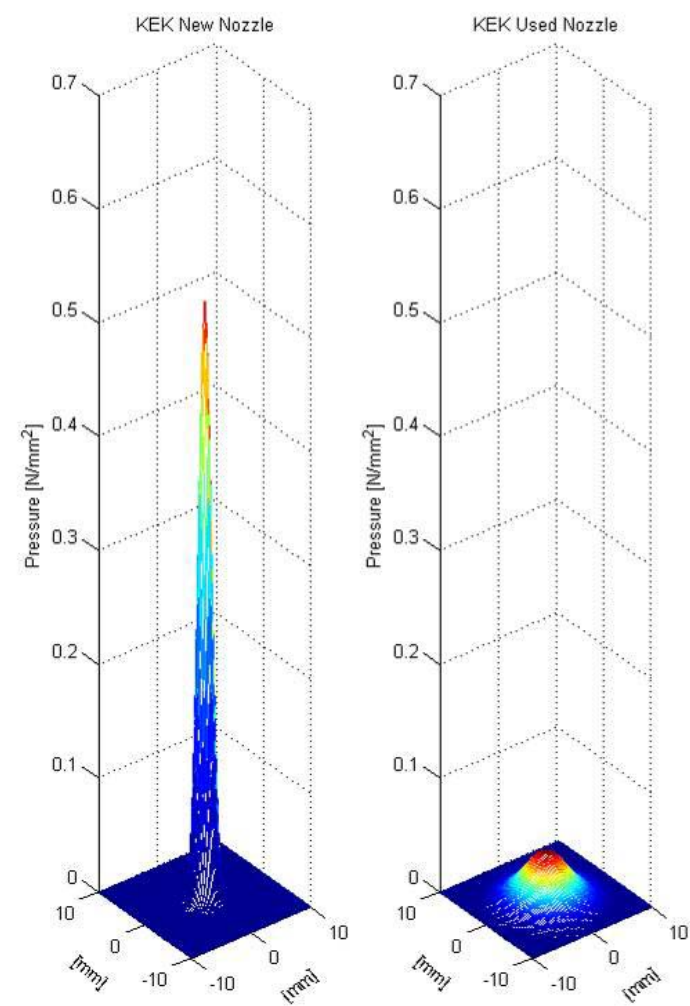

Figure 4: Pressure exerted by the jet on a flat surface at $101 \mathrm{~mm}$ by a new (left) and a used (right) nozzle.

The JLAB HPR process usually employs "fan" jets, generated by nozzles manufactured by Spraying System Co. They produce a solid high throughput "Vee" water jet: the amount of water sprayed by each nozzle is about five times the one of a single nozzle used at KEK.

The "fan" jet is no longer a cylindrically symmetric structure and for its profiling we scan the jet along two perpendicular directions with a target. The value of the force vs. position obtained for each scan, represents the integral of the force along one of the two perpendicular directions. Data can be fitted (Gaussian profile for the edges, plateau for the core) and combined to get the jet profile. Measurements have been done for 3 nozzles: 1502 (fan, $15^{\circ}$ angled), 4002 and 40015 (fan, $40^{\circ}$ angled

T07 Superconducting RF 
but with different throughput) at different distances. Figure 5 shows the measurement principle.

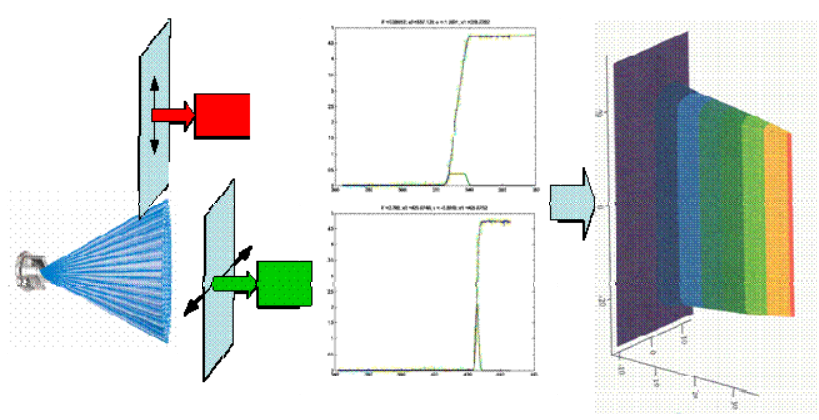

Figure 5: "Fan" jet scanning: two perpendicular scans give the information for the jet profile reconstruction.

The jet profiling, the jet evolution vs. the distance, and the exerted pressure at various nozzle to target distances is shown in Fig. 6 for two different nozzles manufactured by Spraying System Co.: 1502 (15 ${ }^{\circ}$ angled) $4002\left(40^{\circ}\right.$ angled) operated at the same pump pressure (85 bar) and water thoughput.
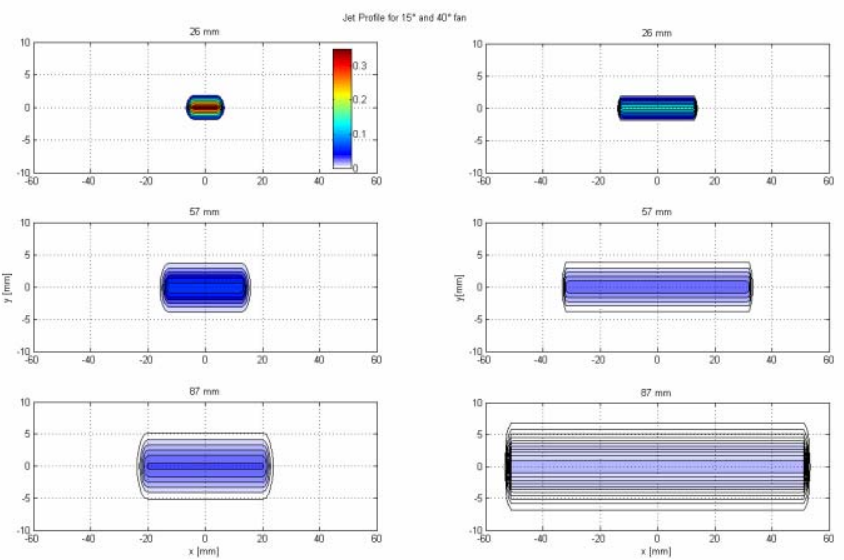

Figure 6: $15^{\circ}$ and $40^{\circ} \mathrm{JLAB}$ "fan" jet profiling at various nozzle to target distances: $26 \mathrm{~mm}, 57 \mathrm{~mm}$ and $87 \mathrm{~mm}$. The pressure unit is $\mathrm{N} / \mathrm{mm}^{2}$.

JLAB measurements analysis indicates that the pressure exerted by the jet produced by "fan" nozzle is well modelled in one direction by a transversal Gaussian structure and on the other one by two Gaussian tails and a flat plateau.

More local information of the jet instabilities can be obtained from the recording of the force transferred on a target smaller than the jet dimensions.

Fig. 7 shows the case of the JLAB fan jet scanned with a $30 \times 30 \mathrm{~mm}^{2}$ target at $165 \mathrm{~mm}$ from the nozzle. The reduced area for the integration of the jet force increases the sensitivity of the diagnostic device. On the contrary the information of the total force transferred to the target is lost.

Therefore these measurements, with the integration on a short target, are complementary with the information obtained during a full intercepting measurement.

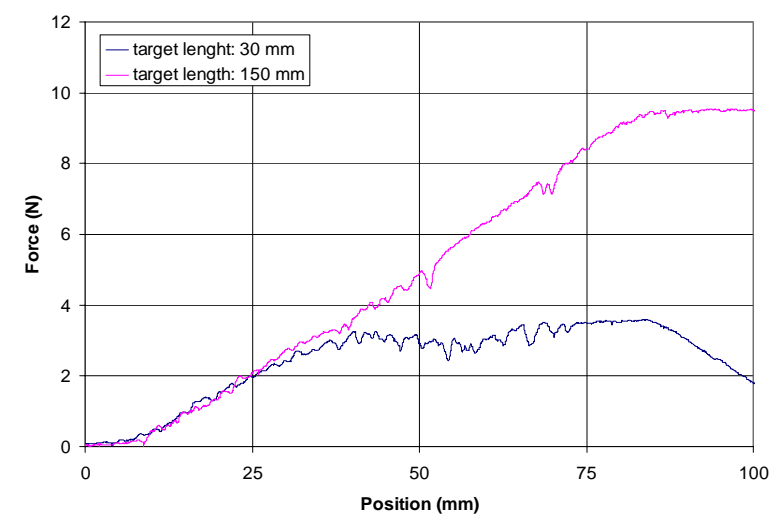

Figure 7: $15^{\circ}$ angled (1502) nozzle jet scanned with a shorter target. The reduced integration area increases the capability to give water jet "local" information.

\section{DISCUSSION AND CONCLUSION}

The measuring device used for the jet characterization developed at INFN Milano - LASA has been used for jet qualification of HPR systems. The device gives information about the stability of the pressure of the high pressure water pump, the water jet structure, the stability of the jet position and the presence of local non uniformities inside the water jet. Moreover the estimation of the local pressure exerted by the jet on the cavity surface can be calculated. The system can qualify round water jets and also not cylindrically symmetric jets as the "fan" used at JLAB.

The investigations done at KEK and Nomura indicated that the jets produced by "used" nozzles generate a local pressure on a cavity surface about one order of magnitude lower than the one from a new nozzle, despite the fact that the water throughput, the pump pressure and the total force exerted by the jet is about the same.

JLAB measurement shows that the pressure exerted by the water jet on the cavity surface is significantly lower then the one produced by jets generated by new KEK nozzles. At the ILC cavity iris distance, the pressure of JLAB jets is about the same as the one generated by the KEK HPR new nozzles at the ILC equator. Nevertheless the water throughput for each nozzle is larger, as larger is the surface covered by the water jet.

The device we have developed allows the fully characterization of HPR water jets. A further step in the optimization of the process requires a qualification of the overall cleaning effect of the jet on the surface. For this, a deeper understanding of the interaction of the jet on the cavity wall is required as well as the experimental qualification of the cleaning efficiency of the jets.

\section{REFERENCES}

[1] P. Kneisel and B. Lewis, Part. Accel. 53 (1996), 97.

[2] R. Gim et al., $4^{\text {th }}$ Symp. On Particle on Surf., p. 379.

[3] E. Cavaliere et al., Physica C, 441 (2006) 254.

[4] R. Reitz, "Fluid Mechanics", CRC Press LLC, 1999.

[5] T. Higo, ILC Asia 2007-01

[6] D. Wright, J. Wolgamott, G. Zink, 2003, WJTA conf. 